\title{
Ortho-bis(amino)arylnickel(II) Halide Complexes Containing Perfluoroalkyl Chains as Model Catalyst Precursors for Use in Fluorous Biphase Systems. ${ }^{\dagger}$
}

\author{
Henk Kleijna , Johann T. B. H. Jastrzebskia, Robert A. Gossage ${ }^{a}$, Huub Kooijmanb, \\ Anthony L. Spek ${ }^{b}$ and Gerard van Koten ${ }^{* a}$ \\ aDebye Institute, Department of Metal-Mediated Synthesis, Utrecht University, Padualaan 8, 3584 CH Utrecht, The Netherlands,
}

bBijvoet Center for Biomolecular Research, Department of Crystal- and Structural Chemistry, Utrecht University, Padualaan 8 , $3584 \mathrm{CH}$ Utrecht, The Netherlands.

*Author to whom correspondence should be addressed; e-mail: vankoten @xray.chem.ruu.nl

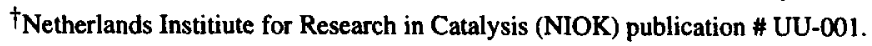

Received 22 July 1997; accepted 12 August 1997

\begin{abstract}
A novel synthetic procedure is presented to modify 1,3-bis[(dimethylamino)methyl]benzene to accommodate perfluoro alkyl chains. These ligands are then converted into nickel-and platinum-chloride organometallic derivatives, which can serve as model compounds for use in homogeneous catalysis. A nickel complex is shown to be an active catalyst for the $1: 1$ addition of $\mathrm{CCl}_{4}$ to methylmethacrylate (Kharasch addition reaction) in dichloromethane but lacks sufficient solubility for use under fluorous biphase conditions. The structure in the solid state of this fluorous $\mathrm{Ni}$ species, which is a rare example of a structurally characterised perfluoro organometallic compound, was elucidated by single crystal X-ray diffraction. (c) 1998 Elsevier Science Ltd. All rights reserved.
\end{abstract}

\section{INTRODUCTION}

When compared to their heterogeneous analogues, homogeneous catalytic systems typically have better product and substrate selectivity characteristics and proceed under milder reaction conditions. ${ }^{1}$ Unfortunately, separation of the dissolved catalyst from the product mixture can be difficult, expensive, and in some cases impossible. ${ }^{1}$ This has led to considerable effort on the design of systems which combine the recovery advantages of heterogeneous conditions with the reactivity properties of isolable homogeneous catalysts. This can be accomplished in a number of ways. For example, the immobilisation of homogeneous catalytic sites onto soluble polymers $^{2}$ or via connection to the periphery of dendrimers ${ }^{3}$ allows the catalyst and its inert support to be readily filtered (i.e. recovered) from the product stream.1,4 A second method that is used to facilitate catalyst recovery involves the use of a biphasic system, usually water and an organic solvent, in which the catalyst is soluble in water but the product(s) are only miscible in the organic layer, e.g., the use of water soluble sulfonated triphenylphosphine $\mathrm{Rh}$ complexes for olefin hydroformylation. ${ }^{1}$ Unfortunately, many homogeneous catalysts are poisoned by the presence of water.

Recently, Horváth and Rábai have developed a new Fluorous Biphase System [FBS] in which a combination of a perfluoro- and an organic-solvent is used and have applied this medium in the Rh catalysed 
hydroformylation of higher alkenes. ${ }^{5}$ These olefins can not readily be converted in a classical aqueous/organic biphasic solvent system. ${ }^{1}$

Several known combinations of perfluoro- and organic-solvents show unusual behaviour and properties. For example, a mixture of perfluoro-methylcyclohexane and octane forms a biphasic system at temperatures below $36.5^{\circ} \mathrm{C}$. Above this temperature, however, a homogeneous solvent mixture is obtained. This allows one to catalyse a reaction under single phase conditions at elevated temperatures and then perform catalyst/product separation under biphasic conditions at room temperature ( $\mathrm{rt}) .{ }^{5}$ As organometallics are generally unreactive towards perfluoro solvents, we became interested in using this novel technology with our own catalyst systems. $2,3,6,7$ In this report we describe our first results in the area of FBS chemistry.

\section{RESULTS}

We have previously shown that aryldiamines 1 (Fig 1; $\mathrm{Y}=\mathrm{H}$ or halogen) are valuable ligand precursors to a number of organometallic complexes that are active in homogeneous catalysis. ${ }^{2,3,6}$ Examples of this include the Kharasch addition of haloalkanes to olefins [1: $\mathrm{Y}=\mathrm{NiX} ; \mathrm{X}=$ halogen], 2,3,6 selective olefin hydrogenation processes and in hydrogen transfer reactions $\left[1: \mathrm{Y}=\mathrm{RuX}\left(\mathrm{PPh}_{3}\right) ; \mathrm{X}=\mathrm{Cl}\right.$ or OTf $] .^{7}$

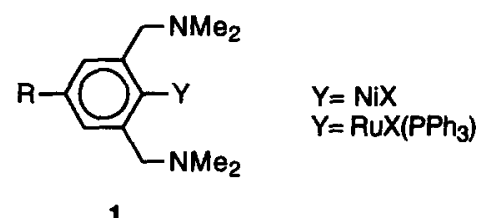

Figure 1

It is thus a challenge to modify amine 1 in such a way that its organometallic derivatives will be suitable for use in a FBS (i.e., soluble in perfluoro- organic solvents). Generally, this involves the synthesis of ligands which contain long chain groups containing a number of perfluoro substituents. Simple modification of ligand 1 with perfluoroalkyl chains at the aromatic nucleus is one obvious approach, but we wished to avoid the strong electron-withdrawing properties of the perfluoroalkyl groups. Electron-withdrawing groups $(R)$ have been previously shown to reduce the catalytic activity of the resulting complexes. ${ }^{8}$ Thus, a spacer group is required which contains at least two, preferably carbon and/or silicon, atoms between the fluoroalkyl groups and the metal-bound aryl ring. Moreover, the fluorous containing building block must contain a suitable reactive functionality for coupling to an aryldiamine. We therefore used perfluoroalkyl chains which terminate with a $\mathrm{CH}_{2} \mathrm{CH}_{2} \mathrm{SiMe}_{2} \mathrm{Cl}$ group as the synthon.

The synthesis of compounds $3 \mathbf{a}$ and $\mathbf{3 b}$ (Scheme 1) is straightforward starting from 5-bromo-1,3bis[(dimethylamino)methyl]benzene (2) ${ }^{9}$ Selective lithiation of 2 was carried out by treatment with two equiv. of $t$ - $\mathrm{BuLi}$ in $\mathrm{Et}_{2} \mathrm{O}$ at $-78^{\circ} \mathrm{C}$ followed by reaction with $(1 \mathrm{H}, 1 \mathrm{H}, 2 \mathrm{H}, 2 \mathrm{H}$-perfluoro-1-octyl)dimethylsilyl chloride or the corresponding ( $1 \mathrm{H}, 1 \mathrm{H}, 2 \mathrm{H}, 2 \mathrm{H}$-perfluoro-1-dodecyl)dimethylsilyl chloride. This procedure afforded the desired ligands in high yields. These compounds were characterised by elemental analysis, ${ }^{1} \mathrm{H},{ }^{13} \mathrm{C}\left\{{ }^{1} \mathrm{H}\right\}$, and ${ }^{19} \mathrm{~F}\left({ }^{1} \mathrm{H}\right\}$ NMR spectroscopy and by mass spectrometry. 


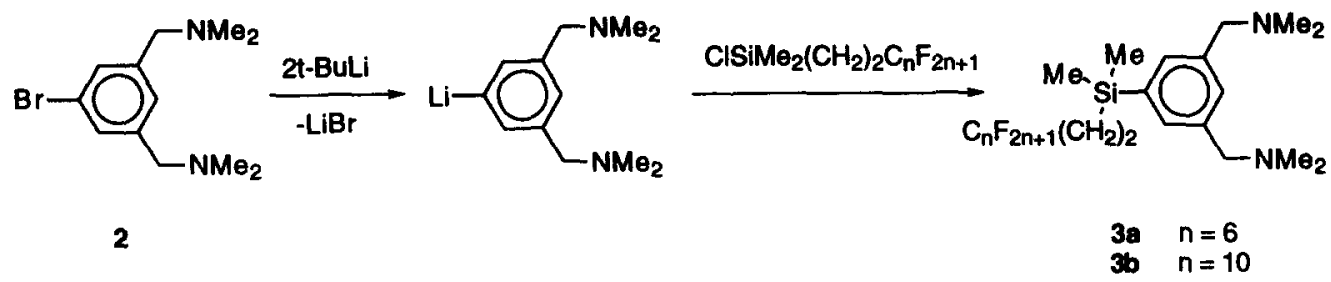

Scheme 1

Ligand precursors $3 \mathbf{a}$ and $\mathbf{3 b}$ (Scheme 2) can be converted into the 2-lithio derivatives via a heteroatom assisted lithiation. Thus, the treatment of $3 \mathbf{a}$ or $3 \mathbf{b}$ with one equiv. of $t-\mathrm{BuLi}$ in pentane at room temperature resulted in the selective and quantitative formation of $4 \mathbf{a}$ and $\mathbf{4 b}$. This was suggested by the reaction with $\mathrm{D}_{2} \mathrm{O}$, in which complete incorporation of deuterium was observed via ${ }^{1} \mathrm{H}$ and ${ }^{13} \mathrm{C}\left\{{ }^{1} \mathrm{H}\right\} \mathrm{NMR}$ and mass spectrometry of the resulting amines. Reaction of in situ prepared $4 \mathrm{a}$ and $4 \mathrm{~b}$ with $\mathrm{PtCl}_{2}\left(\mathrm{SEt}_{2}\right)_{2}$ or $\mathrm{NiCl}_{2}\left(\mathrm{PEt}_{3}\right)_{2}$ afforded compound 5a, 5b, and 6a, 6b, respectively. These novel perfluoralkyl functionalised organometallic complexes were fully characterised spectroscopically and by elemental analysis.

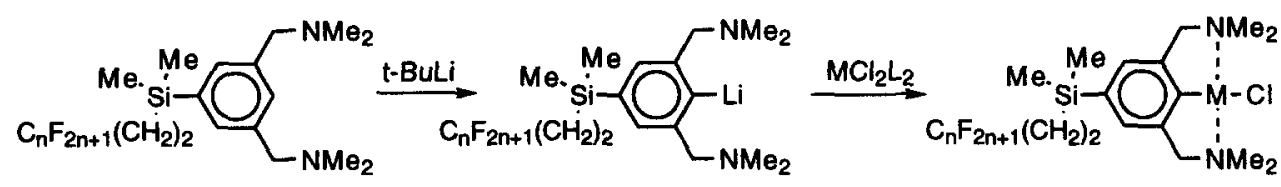

$$
\begin{array}{llll}
3 a & n=6 & 4 a & n=6 \\
3 b & n=10 & 4 b & n=10
\end{array}
$$

Scheme 2

The solid state structure of the nickel compound $6 \mathrm{a}$ was elucidated by single crystal X-ray diffraction and is very similar to the previously characterised 2,6-bis[2-(S)-2-methyl-1-pyrrolidinyl)methyl]phenyl-1-nickel bromide. ${ }^{10}$ Both complexes have an approximately square-planar coordination around the $\mathrm{Ni}$ centre which is $\mathrm{N}, \mathrm{C}, \mathrm{N}^{\prime}$-bonded to the ligand system. The halogen atom occupies the fourth coordination site trans to $\mathrm{C}_{\mathrm{ipso}}$ of the metal-bound aryl ring. The $\mathrm{SiMe}_{2}\left(\mathrm{CH}_{2}\right)_{2} \mathrm{C}_{6} \mathrm{~F}_{13}$ "tail" shows a linear chain structure with the substituents in a staggered conformation. The carbon chain shows a torsion of $30^{\circ}$ over its entire length. An ORTEP diagram of complex 6a appears in Figure 2; a listing of selected bond length and angles is tabulated in Table 1.

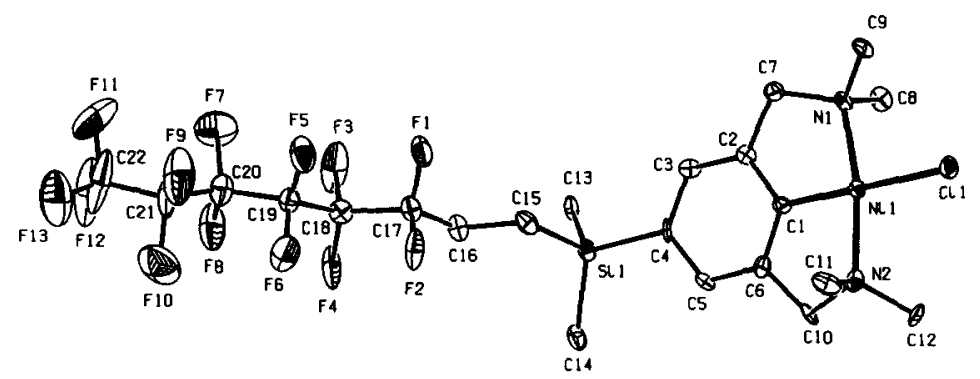

Figure 2. ORTEP drawing of $6 a$ (at the $30 \%$ probability level) showing the adopted numbering scheme. 


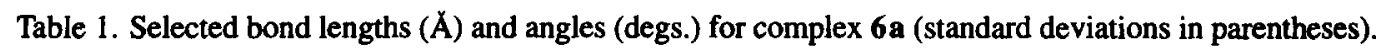

Bond Lengths

$\begin{array}{ll}\text { Ni1-C1 } & 1.823(9) \\ \text { Ni1-Cl1 } & 2.233(3) \\ \text { Ni1-N1 } & 1.983(8) \\ \text { Ni1-N2 } & 1.971(8) \\ \text { Sil-C4 } & 1.876(4)\end{array}$

Bond Angles

$$
\begin{array}{ll}
\text { C1-Ni1-Cl1 } & 179.1(4) \\
\text { N1-Ni1-N2 } & 167.5(3) \\
\text { C4-Si1-C15 } & 106.5(5)
\end{array}
$$

Perfluoro compounds show a great tendency to form waxes and for this reason very few examples of organometallics containing perfluorous tails such as $\mathrm{C}_{6} \mathrm{~F}_{13}$ have been crystallographically characterised. ${ }^{11}$ Our attempts to grow suitable crystals of $\mathbf{6 b}$ were probably unsuccessful for this reason. The new nickel compounds 6a and $6 \mathrm{~b}$ were tested also in a standard Kharasch addition reaction of $\mathrm{CCl}_{4}$ to methylmethacrylate in dichloromethane solution (Scheme 3) following the standard protocols developed in our earlier studies. $2,3,6,8$

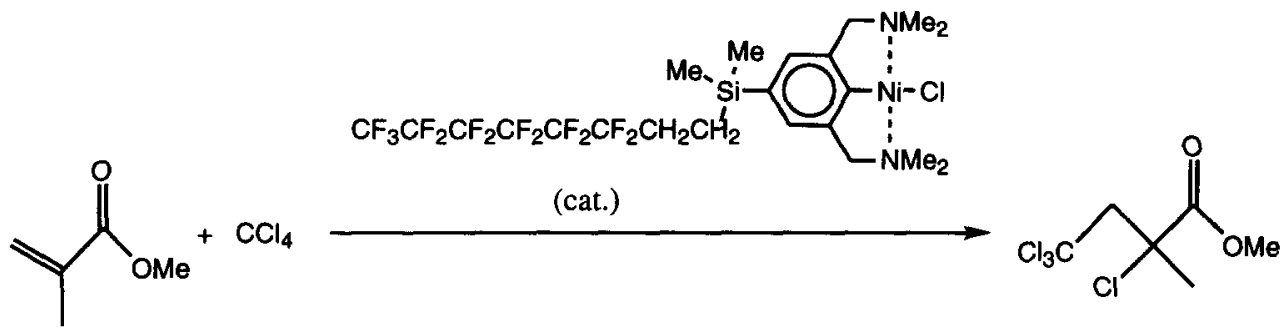

Scheme 3

The results were then compared with those of the parent compound 1 (Fig. $1 ; \mathrm{Y}=\mathrm{NiBr}, \mathrm{R}=\mathrm{H}$ ). ${ }^{3,8 \mathrm{~b}}$ All three nickel compounds show almost identical catalytic activity; i.e. similar reaction rates as well as complete selectivity towards the formation of the $1: 1$ addition product. The mechanism of this reaction has been previously studied and involves $\mathrm{Ni}$-mediated haloalkane activation followed by single electron and halide transfer to the alkene. Reaction of the olefin is the rate determining step in this chemistry. ${ }^{3 b, 8 b}$ Unfortunately, neither compound 6a nor $6 \mathrm{~b}$ was found to be of sufficient solubility (UV/Vis. spectroscopy) ${ }^{8 a}$ for use in a FBS experiment. Thus, these compounds can only serve as model complexes in terms of their spectroscopic features, catalytic activity and the synthetic protocol used in their production. For improved solubility of perfluoro organometallics in perfluoro solvents, it is known that instead of one perfluorotail, that often three perfluoro tails (ponytails) are necessary to obtain good partition characteristics over the two phases. ${ }^{5,12}$ We are currently applying the synthetic techniques described herein to attach such ponytails to our bis-ortho-(diamino)aryl ligand systems.

\section{CONCLUDING REMARKS}

We have demonstrated an effective procedure for the synthesis of perfluoro-modified bis-ortho-(diamino)aryl ligand precursors 3 . These compounds have been shown to produce the corresponding $\mathrm{Ni}$ and $\mathrm{Pt}$ organometallic 
complexes in a facile manner from readily available starting materials. In addition, the $\mathrm{Ni}$ complexes have shown very similar catalytic behaviour when compared to the non-fluorous modified materials. Specifically, this catalysis involves the $1: 1$ Kharasch addition of polyhalogenated alkanes to olefins. The presence of a single perfluoro "tail" is not effective in facilitating solubility of the organometallic derivatives in a Fluorous Biphase System.

\section{EXPERIMENTAL SECTION}

General. All synthetic manipulations with air-sensitive reagents were carried out under a dry, dinitrogen atmosphere using standard Schlenk techniques. Solvents were dried and distilled by literature methods immediately prior to use. The complex $\mathrm{PtCl}_{2}\left(\mathrm{SEt}_{2}\right)_{2}$ and $\mathrm{NiCl}_{2}\left(\mathrm{PEt}_{3}\right)_{2}$ were prepared by literature methods. ${ }^{13,14}$ All other chemicals were purchased commercially. The instrumentation used in this work was identical to that previously described. ${ }^{8 a}$ All NMR chemical shifts are referenced to TMS $\left({ }^{1} \mathrm{H}\right.$ and $\left.{ }^{13} \mathrm{C}\left({ }^{1} \mathrm{H}\right\}\right)$ or $\mathrm{CFCl}_{3}$ $\left({ }^{19} \mathrm{~F}\left\{{ }^{1} \mathrm{H}\right\}\right)$. General directions concerning the catalytic testing of complexes $6 a$ and $6 \mathrm{~b}$ can be found in reference 8.

Preparation of 5-[(tridecafluoro-1,1,2,2-tetrahydrooctyl)-1-dimethylsilyl]-1,3[(dimethylamino)methyl]benzene (3a): To a stirred solution of $2.7 \mathrm{~g}(10 \mathrm{mmol})$ of $2^{9}$ in hexanes (10 mL) at $-70^{\circ} \mathrm{C}$ was added $t-\mathrm{BuLi}(13.3 \mathrm{~mL}, 1.5 \mathrm{M}$, pentane). The reaction mixture was allowed to raise to $\mathrm{rt}$ after which stirring was continued for $30 \mathrm{~min}$. Subsequently, $4.4 \mathrm{~g}(10 \mathrm{mmol})$ of (tridecafluoro-1,1,2,2tetrahydrooctyl)-1-dimethylsilyl chloride was added. The contents were then stirred overnight. The resulting mixture was then poured into water and the aqueous portion extracted twice with pentane $(25 \mathrm{~mL})$. The combined organic layers were dried $\left(\mathrm{Na}_{2} \mathrm{SO}_{4}\right)$, filtered and concentrated to yield $5.53 \mathrm{~g}(92 \%)$ of crude product. The product was further purified by shortpath distillation; bp $135-140^{\circ} \mathrm{C} / 0.5 \mathrm{~mm} \mathrm{Hg} ;{ }^{1} \mathrm{H} \mathrm{NMR},\left(\mathrm{CDCl}_{3}\right): \delta 7.46$ (s, $2 \mathrm{H}$, aromatic ortho $H) ; 7.27(\mathrm{~s}, 1 \mathrm{H}$, aromatic $H) ; 3.41\left(\mathrm{~s}, 4 \mathrm{H}, \mathrm{CH}_{2} \mathrm{~N}\right) ; 2.17\left(\mathrm{~s}, 12 \mathrm{H}, \mathrm{NCH}_{3}\right) ; 2.06-1.88(\mathrm{~m}$, $\left.2 \mathrm{H}, \mathrm{CF}_{2} \mathrm{CH}_{2}\right) ; 1.00-0.84\left(\mathrm{~m}, 2 \mathrm{H}, \mathrm{CH}_{2} \mathrm{Si}\right) ; 0.32\left(\mathrm{~s}, 6 \mathrm{H}, \mathrm{SiCH}_{3}\right) ;{ }^{13} \mathrm{C}\left\{{ }^{1} \mathrm{H}\right\} \mathrm{NMR},\left(\mathrm{CDCl}_{3}\right): \delta 138.6,137.2$, 133.2 and 131.4 (aromatic $C$ ), $64.6\left(\mathrm{CH}_{2} \mathrm{~N}\right) ; 45.5\left(\mathrm{NCH}_{3}\right) ; 26.2\left(\mathrm{t}, \mathrm{J}_{\mathrm{F}-\mathrm{C}}=24 \mathrm{~Hz}, \mathrm{CF}_{2} \mathrm{CH}_{2}\right) ; 5.5\left(\mathrm{SiCH}_{2}\right) ;-3.2$ $\left(\mathrm{SiCH}_{3}\right) ;{ }^{19} \mathrm{~F}\left({ }^{1} \mathrm{H}\right\}$ NMR $\left(\mathrm{C}_{6} \mathrm{D}_{6}\right): \delta-81.2(3 F),-115.6(2 F),-122.0(4 F),-123.2(2 F),-126.3(2 F)$. MS $(70$ eV): $\mathrm{M}^{+}$596. Anal. calcd. for $\mathrm{C}_{22} \mathrm{H}_{29} \mathrm{~F}_{13} \mathrm{~N}_{2} \mathrm{Si}$ : C 44.29, H 4.90, N 4.70, Si 4.71; found: C 44.31, H 5.06, N 4.66, Si 4.78 .

Preparation of 5-[(henicofluoro-1,1,2,2-tetrahydrododecyl)-1-dimethylsilyl] 1,3bis(dimethylaminomethyl)benzene (3b): This compound was synthesized according to the procedure described for 3a using the appropriate silyl chloride. After short path distillation, a white solid was obtained; 98 $\%$; bp $160-180^{\circ} \mathrm{C} / 0.5 \mathrm{~mm} \mathrm{Hg}$. ' $\mathrm{H} \mathrm{NMR},\left(\mathrm{CDCl}_{3}\right) ; \delta 7.30$ (s, 2H, aromatic ortho $H$ ); 7.28 (s, 1H, aromatic $H$ ); $3.42\left(\mathrm{~s}, 4 \mathrm{H}, \mathrm{CH}_{2} \mathrm{~N}\right) ; 2.22\left(\mathrm{~s}, 12 \mathrm{H}, \mathrm{NCH}_{3}\right) ; 2.06-1.94\left(\mathrm{~m}, 2 \mathrm{H}, \mathrm{CF}_{2} \mathrm{CH}_{2}\right) ; 1.00-0.89\left(\mathrm{~m}, 2 \mathrm{H}, \mathrm{CH}_{2} \mathrm{Si}\right) ; 0.32$ (s, $\left.6 \mathrm{H}, \mathrm{SiCH}_{3}\right) ;{ }^{13} \mathrm{C}\left({ }^{1} \mathrm{H}\right\} \mathrm{NMR}\left(\mathrm{CDCl}_{3}\right): \delta 138.3,136.0,133.9$ and 131.2 (aromatic $\mathrm{C}$ ); $64.3\left(\mathrm{CH}_{2} \mathrm{~N}\right) ; 45.2$ $\left(\mathrm{NCH}_{3}\right) ; 25.9\left(\mathrm{t}, \mathrm{J}_{\mathrm{F}-\mathrm{C}}=24 \mathrm{~Hz}, \mathrm{CF}_{2} \mathrm{CH}_{2}\right) ; 5.2\left(\mathrm{SiCH}_{2}\right) ;-3.5\left(\mathrm{SiCH}_{3}\right) ;{ }^{19} \mathrm{~F}\left\{{ }^{1} \mathrm{H}\right\} \mathrm{NMR}\left(\mathrm{C}_{6} \mathrm{D}_{6}\right): \delta-81.3(3 F)$, $115.7(2 F),-121.7(10 F),-122.9(2 F),-123.2(2 F),-126.4(2 F)$. MS $(70 \mathrm{eV}): \mathrm{M}^{+} 796$. Anal. calcd. for $\mathrm{C}_{26} \mathrm{H}_{29} \mathrm{~F}_{21} \mathrm{~N}_{2} \mathrm{Si}$ : C 39.20, H 3.67, N 3.52, Si 3.53; found: C 39.08, H 3.64, N 3.55, Si 3.57. 
Preparation of 4-[(tridecafluoro-1,1,2,2-tetrahydrooctyl)-1-dimethylsilyl]-2,6bis[(dimethylamino)methyl]phenyl-1-platinum chloride (5a): To a stirred solution of $3 \mathrm{a}(1.4 \mathrm{~g}, 2.3$ $\mathrm{mmol})$ in hexane $(10 \mathrm{~mL})$, was added a pentane solution of $t$-BuLi $(1.5 \mathrm{~mL}, 1.5 \mathrm{M})$. After stirring for $0.5 \mathrm{~h}$., bis(diethylsulfide)platinum dichloride $(1 \mathrm{~g}, 2.2 \mathrm{mmol})$ in THF $(10 \mathrm{~mL})$ was added. The reaction was then stirred overnight at $\mathrm{rt}$. The organic solvents were evaporated in vacuo and the residue dissolved in warm benzene ( 25 $\mathrm{mL}$ ). After rapid filtration through filter paper, the benzene was evaporated and the solid thus obtained was washed with pentane. The product was isolated as a brown solid which contained traces of $\operatorname{Pt}(0)$. These impurities can be removed by treating the product in hot ethanol with activated carbon; ${ }^{1} \mathrm{H} N \mathrm{NM}\left(\mathrm{C}_{6} \mathrm{D}_{6}\right): \delta 6.80$ (s, 2H, aromatic $H$ ); $3.35\left(\mathrm{t}, 4 \mathrm{H}, \mathrm{J}_{\mathrm{Pt}-\mathrm{H}}=22.2 \mathrm{~Hz}, \mathrm{CH}_{2} \mathrm{~N}\right) ; 2.71\left(\mathrm{t}, 12 \mathrm{H}, \mathrm{J}_{\mathrm{Pt}-\mathrm{H}}=18.8 \mathrm{~Hz}, \mathrm{NCH}_{3}\right) ; 2.16-1.90$ $\left(\mathrm{m}, 2 \mathrm{H}, \mathrm{CF}_{2} \mathrm{CH}_{2}\right) ; 1.01-0.96\left(\mathrm{~m}, 2 \mathrm{H}, \mathrm{CH}_{2} \mathrm{Si}\right) ; 0.19(\mathrm{~s}, 6 \mathrm{H}, \mathrm{SiCH}) ;{ }^{13} \mathrm{C}\left\{{ }^{1} \mathrm{H}\right\}$ NMR $\left(\mathrm{C}_{6} \mathrm{D}_{6}\right): \delta 150.4\left(\mathrm{t}, \mathrm{J}_{\mathrm{Pt}-\mathrm{C}}\right.$ $=477 \mathrm{~Hz}$, ipso $C) ; 144.1\left(\mathrm{t}, \mathrm{J}_{\mathrm{Pt}-\mathrm{C}}=38 \mathrm{~Hz}\right.$, aromatic $\left.C\right) ; 130.0($ aromatic $C) ; 123.9\left(\mathrm{t}, \mathrm{J}_{\mathrm{Pt}-\mathrm{C}}=17 \mathrm{~Hz}\right.$, aromatic C); $77.7\left(\mathrm{t}, \mathrm{J}_{\mathrm{Pt}-\mathrm{C}}=32 \mathrm{~Hz}, \mathrm{CH}_{2} \mathrm{~N}\right) ; 54.0\left(\mathrm{NCH}_{3}\right) ; 26.5\left(\mathrm{t}, \mathrm{J}_{\mathrm{F}-\mathrm{C}}=24 \mathrm{~Hz}, \mathrm{CF}_{2} \mathrm{CH}_{2}\right) ; 5.6(\mathrm{SiCH}) ;-3.2\left(\mathrm{SiCH}_{3}\right)$; ${ }^{19} \mathrm{~F}\left\{{ }^{1} \mathrm{H}\right\}$ NMR $\left(\mathrm{C}_{6} \mathrm{D}_{6}\right): \delta-80.9(3 F),-115.3(2 F),-121.7(4 F),-122.8(2 F),-126.0(2 F)$. Anal. calcd. for $\mathrm{C}_{22} \mathrm{H}_{28} \mathrm{ClF}_{13} \mathrm{~N}_{2} \mathrm{PtSi}$ : C 31.99, H 3.42, N 3.39, Si 3.40; found: C 32.09, H 3.48, N 3.31, Si 3.52.

Preparation of 4-[(henicofluoro-1,1,2,2-tetrahydrododecyl)-1-dimethylsilyl]-2,6[(dimethylamino)methyl]phenyl-1-platinum chloride (5b): The product was synthesized according to the method described for complex $5 \mathrm{a}$ using the appropriate ligand precursor $3 \mathrm{~b}$. Yield: $82 \%$; ${ }^{1} \mathrm{H} \mathrm{NMR}\left(\mathrm{CDCl}_{3}\right)$ : $\delta 6.70(\mathrm{~s}, 2 \mathrm{H}$, aromatic $H) ; 4.04\left(\mathrm{t}, 4 \mathrm{H}, \mathrm{J}_{\mathrm{Pt}-\mathrm{H}}=22.7 \mathrm{~Hz}, \mathrm{CH}_{2} \mathrm{~N}\right) ; 3.09\left(\mathrm{t}, 12 \mathrm{H}, \mathrm{J}_{\mathrm{Pt}-\mathrm{H}}=17.1 \mathrm{~Hz}, \mathrm{NCH}_{3}\right)$; 2.17-1.87 (m, 2H, $\left.\mathrm{CF}_{2} \mathrm{CH}_{2}\right) ; 0.97-0.89\left(\mathrm{~m}, 2 \mathrm{H}, \mathrm{CH}_{2} \mathrm{Si}\right) ; 0.27\left(\mathrm{~s}, 6 \mathrm{H}, \mathrm{SiCH}_{3}\right) ;{ }^{13} \mathrm{C}\left({ }^{1} \mathrm{H}\right\} \mathrm{NMR}\left(\mathrm{CDCl}_{3}\right): \delta$ $147.6\left(\mathrm{t}, \mathrm{J}_{\mathrm{Pt}-\mathrm{C}}=477 \mathrm{~Hz}\right.$, ipso $\left.C\right) ; 143.4\left(\mathrm{t}, \mathrm{J}_{\mathrm{Pt}-\mathrm{C}}=38 \mathrm{~Hz}\right.$, aromatic $\left.C\right) ; 131.0($ aromatic $C) ; 124.0\left(\mathrm{t}, \mathrm{J}_{\mathrm{Pt}-\mathrm{C}}=\right.$ $17 \mathrm{~Hz}$, aromatic $C) ; 77.7\left(\mathrm{t}, \mathrm{J}_{\mathrm{Pt}-\mathrm{C}}=32 \mathrm{~Hz}, \mathrm{CH}_{2} \mathrm{~N}\right) ; 54.4\left(\mathrm{NCH}_{3}\right) ; 26.0\left(\mathrm{t}, \mathrm{J}_{\mathrm{F}-\mathrm{C}}=24 \mathrm{~Hz}, \mathrm{CF}_{2} \mathrm{CH}_{2}\right) ; 5.3$ $\left(\mathrm{SiCH}_{2}\right) ;-3.3\left(\mathrm{SiCH}_{3}\right) ;{ }^{19} \mathrm{~F}\left\{{ }^{1} \mathrm{H}\right\}$ NMR $\left(\mathrm{CDCl}_{3}\right): \delta-80.8(3 F) ;-115.8(2 F) ;-121.8(10 F) ;-122.7(2 F) ;-123.1$ $(2 F)$; -126.1 (2F). Anal. calcd. for $\mathrm{C}_{22} \mathrm{H}_{28} \mathrm{ClF}_{13} \mathrm{~N}_{2} \mathrm{PtSi}$ : C 30.43, H 2.75, N 2.73, Si 2.74; found: C $30.35, \mathrm{H}$ 2.79, N 2.72, Si 2.79 .

Preparation of 4-[(tridecafluoro-1,1,2,2-tetrahydrooctyl)-1-dimethylsilyl]-2,6[(dimethylamino)methyl]phenyl-1-nickel chloride (6a): To a stirred solution of $3 \mathrm{a}(1.1 \mathrm{~g}, 1.8 \mathrm{mmol})$ in hexane $(10 \mathrm{~mL})$, was added $t-B u L i(1.2 \mathrm{~mL}, 1.5 \mathrm{M})$. The reaction mixture was stirred for $0.5 \mathrm{~h}$., then bis(triethylphosphine)nickel dichloride $(0.66 \mathrm{~g}, 1.8 \mathrm{mmol}$ ) was added after which the red color changed directly to orange-yellow. After overnight stirring, the solid was separated from the solvent by centrifugation and then washed 3 times with pentane. The yellow solid was then dissolved in benzene and centrifuged to remove lithium chloride. The benzene solution was subsequently concentrated to yield $1 \mathrm{~g}(83 \%)$ of the pure product; ${ }^{1} \mathrm{H}$ NMR $\left(\mathrm{C}_{6} \mathrm{D}_{6}\right): \delta 6.62(\mathrm{~s}, 2 \mathrm{H}$, aromatic $\mathrm{H}) ; 3.07\left(\mathrm{~s}, 4 \mathrm{H}, \mathrm{CH}_{2} \mathrm{~N}\right) ; 2.40\left(\mathrm{~s}, 12 \mathrm{H}, \mathrm{NCH}_{3}\right) ; 2.16-1.94\left(\mathrm{~m}, 2 \mathrm{H}, \mathrm{CF}_{2} \mathrm{CH}_{2}\right)$; 1.08-0.92 (m, 2H, $\left.\mathrm{CH}_{2} \mathrm{Si}\right) ; 0.16\left(\mathrm{~s}, 6 \mathrm{H}, \mathrm{SiCH}_{3}\right) ;{ }^{13} \mathrm{C}\left\{{ }^{1} \mathrm{H}\right\}$ NMR $\left(\mathrm{C}_{6} \mathrm{D}_{6}\right): \delta 155.6,147.3,132.2$ and 123.3 (aromatic C); $73.7\left(\mathrm{CH}_{2} \mathrm{~N}\right) ; 51.0\left(\mathrm{NCH}_{3}\right) ; 26.4\left(\mathrm{t}, \mathrm{J}_{\mathrm{F}-\mathrm{C}}=24 \mathrm{~Hz}, \mathrm{CF}_{2} \mathrm{CH}_{2}\right) ; 5.7\left(\mathrm{SiCH}_{2}\right) ;-3.2(\mathrm{SiCH})$; ${ }^{19} \mathrm{~F}\left\{{ }^{1} \mathrm{H}\right\}$ NMR $\left(\mathrm{C}_{6} \mathrm{D}_{6}\right): \delta-80.9(3 F) ;-115.3(2 F) ;-121.7(2 F) ; 122.8(4 F) ;-126.0(2 F)$. Anal. calcd. for $\mathrm{C}_{22} \mathrm{H}_{28} \mathrm{ClF}_{13} \mathrm{~N}_{2} \mathrm{NiSi}$ : C 38.31, $\mathrm{H} 4.09, \mathrm{~N} 4.06$; found: C 38.41, H 4.15, N 3.96.

Preparation of 4-[(henicofluoro-1,1,2,2-tetrahydrododecyl)-1-dimethylsilyl]-2,6[(dimethylamino)methyl]phenyl-1-nickel chloride (6b): The product was synthesized according to the 
method described for complex $6 \mathrm{a}$ using the ligand precursor $3 \mathrm{~b} ; 75 \%$; ${ }^{1} \mathrm{H}$ NMR $\left(\mathrm{C}_{6} \mathrm{D}_{6}\right): \delta 6.63$ (s, $2 \mathrm{H}$, aromatic H); 3.10 (s, 4H, $\mathrm{CH}_{2} \mathrm{~N}$ ); 2.41 (s, $\left.12 \mathrm{H}, \mathrm{NCH}_{3}\right) ; 2.19-1.92\left(\mathrm{~m}, 2 \mathrm{H}, \mathrm{CF}_{2} \mathrm{CH}_{2}\right) ; 1.01-0.92\left(\mathrm{~m}, 2 \mathrm{H}, \mathrm{CH}_{2} \mathrm{Si}\right) ; 0.17$ (s, 6H, $\left.\left.\mathrm{SiCH}_{3}\right) .{ }^{13} \mathrm{C}\left({ }^{1} \mathrm{H}\right\}\right) \mathrm{NMR}\left(\mathrm{C}_{6} \mathrm{D}_{6}\right): \delta 155.6,147.3,132.3$ and 123.2 (aromatic $C$ ); $73.8\left(\mathrm{CH}_{2} \mathrm{~N}\right) ; 51.0$ $\left(\mathrm{NCH}_{3}\right) ; 26.6\left(\mathrm{t}, \mathrm{J}_{\mathrm{F}-\mathrm{C}}=24 \mathrm{~Hz}, \mathrm{CF}_{2} \mathrm{CH}_{2}\right) 5.8\left(\mathrm{SiCH}_{2}\right) ;-3.3\left(\mathrm{SiCH}_{3}\right) .{ }^{19} \mathrm{~F}\left({ }^{1} \mathrm{H}\right) \mathrm{NMR}\left(\mathrm{C}_{6} \mathrm{D}_{6}\right): \delta-80.9(3 F)$; $114.9(2 F) ;-121.4(10 F) ;-122.6(4 F) ;-125.7(2 F)$. Anal. calc. for $\mathrm{C}_{26} \mathrm{H}_{28} \mathrm{ClF}_{21} \mathrm{~N}_{2} \mathrm{NiSi}$ : C 35.10, H 3.17, N 3.15, Si 3.16; found: C 35.26, H 3.23, N 3.24, Si 3.19 .

Crystal Structure Determination of Complex 6a: $\mathrm{C}_{22} \mathrm{H}_{28} \mathrm{ClF}_{13} \mathrm{~N}_{2} \mathrm{NiSi}, \mathrm{M}_{\mathrm{r}}=689.69$, yellow, needle-shaped crystal ( $0.05 \times 0.05 \times 0.25 \mathrm{~mm}$ ), monoclinic, space group P2 $1 \mathrm{c}$ (no. 14) with $a=5.9623(6), b=$ 12.2472(17), $c=39.868(5) \AA, \beta=91.805(9)^{\circ}, V=2909.8(6), Z=4, D_{x}=1.5744(3) \mathrm{g} \mathrm{cm}^{-3}, \mathrm{~F}(000)=1400$, $\mu(\mathrm{Mo} \mathrm{K} \alpha)=9.0 \mathrm{~cm}^{-1} .5116$ independent reflections, $\left(0.5^{\circ}<\theta<25.0^{\circ}, \omega\right.$ scan, $T=150 \mathrm{~K}$, Mo K $\alpha$ radiation, $\lambda=0.71073 \AA$ ) measured on a Enraf-Nonius CAD4 Turbo diffractometer on rotating anode. The structure was solved by automated Patterson methods (DIRDIF96). Refinement on $F^{2}$ was carried out by full-matrix leastsquares techniques (SHELXL-96). Hydrogen atoms were included in the refinement on calculated positions riding on their carrier atoms. Refinement converged at a final $w R 2$ value of $0.1899, w=1 /\left[\sigma^{2}\left(F^{2}\right)+\right.$ $\left.(0.0508 \mathrm{P})^{2}\right]$, where $\mathrm{P}=\left(\max \left(\mathrm{F}_{\mathrm{o}}{ }^{2}, 0\right)+2 \mathrm{~F}_{\mathrm{o}}{ }^{2}\right) / 3, R 1=0.0843$ (for $\left.1990 \mathrm{I}>2 \sigma(I)\right), S=0.978$, for 367 parameters. A final difference Fourier showed no residual density outside -0.50 and $0.66 \mathrm{e} \AA^{-3}$.

Crystallographic data (excluding structure factors) have been deposited with the Cambridge Crystallographic Data Centre. Copies of the data can be obtained free of charge on application to The Director, CCDC, 12 Union Road, Cambridge, CB2 1EZ, UK.

Acknowledgements: The authors wish to thank Dr. E. Rijnberg and Mr. P. van Cranenbroek for their contributions to this research. This work is supported by the Netherlands Foundationfor Scientific Research (SON) with financial aid from the Netherlands Organisation for Scientific Research (NWO).

\section{REFERENCES}

1. a: Moulijn, J. A.; van Leeuwen, P. W. N. M.; van Santen, R. A. (eds.) Catalysis: An Integrated Approach to Homogeneous, Heterogeneous and Industrial Catalysis; Elsevier: Amsterdam, 1993; b. Weber, W. P.; Gokel, G. W. Phase Transfer Catalysis in Organic Synthesis; Springer-Verlag: NewYork, 1977; c: Rousseau, R. W. Handbook of Separation Process Technology; John Wiley \& Sons: New York, 1987.

2. van de Kuil, L. A.; Grove, D. M.; Zwikker, J. W.; Jenneskens, L. W.; Drenth, W.; van Koten, G. Chem. Mater. 1994, 6, 1675-1683.

3. Knapen, J. W. J.; van der Made, A. W.; Wilde, J. C.; van Leeuwen, P. W. N. M.; Wijkens, P.; Grove, D. M.; van Koten, G. Nature 1994, 372, 659-663.

4. a: Knapen, J. W. J.; van der Made, A. W.; de Wilde, J. C.; van Leeuwen, P. W. N. M.; Wijkens, P.; Grove, D. M.; Gossage, R. A.; Kragl, U.; Brinkmann, N.; van Koten, G. manuscript in preparation; ${ }^{\text {b. }}$ Kleij, A. W.; Gossage, R. A.; Kleijn, H.; van Koten, G.; Kragl, U.; Brinkmann, N. KNCV Katalyse Symposium, May 12-13, 1997; Kerkrade, The Netherlands, poster 37.

5. Horváth, I. T.; Rábai, J. Science 1994, 266, 72-75.

6. a: Grove, D. M.; Verschuuren, A. H. M.; van Koten, G.; van Beek, J. A. M. J. Organomet. Chem. 1989 , 
372, C1-C6; b: Grove, D. M.; van Koten, G.; Verschuuren, A. H. M. J. Mol. Catal. 1988, 45, 169-174.

7. a: Jiang, Q.; van Plew, D.; Murtuza, S.; Zhang, X. Tetrahedron Lett. 1996, 37, 797-801 and references therein; b: Abbenhuis, R. A. T.; Bergshoef, M. M.; Boersma, J.; Smeets, W. J. J.; Spek, A. L.; van Koten, G., Inorg. Chem., in press; ${ }^{c}$ : Dani, P.; Karlen, T.; van Koten, G., manuscript in preparation.

8. a: van de Kuil, L. A.; Luitjes, H.; Grove, D. M.; Zwikker, J. W.; van der Linden, J. G. M.; Roelofsen, A. M.; Jenneskens, L. W.; Drenth, W.; van Koten, G. Organometallics, 1994, 13, 468-477; b: van de Kuil, L. A.; Grove, D. M.; Gossage, R. A.; Zwikker, J. W.; Jenneskens, J. W.; Drenth, W.; van Koten, G. ibid, 1997, in press.

9. Steenwinkel, P.; James, S. J.; Grove, D. M.; Veldman, N.; Spek, A. L.; van Koten, G. Chem. Eur. J. 1996, 2, 1440-1445.

10. van de Kuil, L. A.; Veldhuizen, Y. S. J.; Grove, D. M.; Zwikker, J. W.; Jenneskens, L. W.; Drenth, W.; Smeets, W. J. J.; Spek, A. L.; van Koten, G. Recl. Trav. Chim. Pays-Bas 1994, 113, 267-277. For related examples see: Grove, D. M.; van Koten, G.; Ubbels, H. J. C.; Zoet, R.; Spek, A. L. Organometallics 1984, 3, 1003-1009; van Beek, J. A. M.; van Koten, G.; Ramp, M. J.; Coenjaarts, N. C.; Grove, D. M.; Goubitz, M. C.; Stam, C. H.; Smeets, W. J. J.; Spek, A. L. Inorg. Chem. 1991, 30, 3059-3068 and Schimmelpfennig, U.; Zimmering, R.; Scheinitz, K. D.; Stoßer, R.; Wenschuh, E.; Baumeister, U.; Hartung, H. Z. Anorg. Allg. Chem. 1991, 619, 1931-1938.

11. a: Kromm, P.; Bideau, J. -P.; Cotrait, M.; Destrade, C.; Nguyen, H. Acta Cryst. Sect. C. 1994, 50, 112115; b: Jablonski, C. R.; Zhou, Z. Can. J. Chem. 1992, 70, 2544-2551.

12. Gladysz, J. A. Science 1994, 266, 55-56.

13. Kauffman, G. B.; Cowan, D. O. Inorg. Syn. 1960, 21, 211-215.

14. Jensen, K. A.; Nielsen, P. H.; Pedersen, C. Th. Acta Chem. Scand. 1963, 17, 1115-1123 and references therein. 